\title{
Incertitudes de l'information et décisions en situation de risque. Cas de problèmes de protection contre les crues
}

\section{Informational and decisional uncertainty factors in hazardous situations as applicable to flood-protection problems}

\author{
Jacques Bernier et Jacques Miquel \\ Électricité de France - Laboratoire National d'Hydraulique
}

\section{Introduction}

L'essentiel des travaux dont cette note fait la synthèse a fait l'objet de contrats passés en 1973 et 1974 entre l'ex-Ministère de l'Equipement et du Logement (Service Central Hydrologique) et le Laboratoire National d'Hydraulique. Le propos de ces travaux était d'examiner les interactions entre les incertitudes de diverses sortes, notamment hydrologiques et économiques, et les décisions dans le domaine de la protection contre les crues. Comme dans les autres études analogues faisant l'objet de contrats semblables, les objectifs étaient essentiellement méthodologiques.

Dans la suite nous considérons la classe des problèmes de dimensionnement où la variable de décision du projecteur est unidimensionnelle, caractérisée généralement par le débit d'une "crue de projet". On sait que l'approche traditionnelle conduit à déterminer le débit maximal annuel dont la probabilité de dépassement $p$ est forfaitairement fixée à une valeur choisie arbitrairement ou par référence à des conséquences dommageables estimées souvent de façon assez floue. Dans cette situation de risque face à l'occurrence d'événements rares (les crues extrêmes), il se pose un premier problème concernant l'interprétation concrète, opérationnelle de cette notion théorique de probabilité de dépassement $p$.

Concernant les incertitudes de toutes sortes sur les données disponibles (données sur les conséquences en particulier économiques du choix d'une crue de projet, données hydrométriques, pluviométriques, géomorphologiques sur les bassins, etc...), le projecteur a pris une conscience plus nette du rôle de ces incertitudes, au moins de certaines d'entre elles, depuis que l'hydrologue lui a fourni une fourchette, un intervalle de confiance sur les crues estimées et non plus une valeur unique. Cependant l'embarras du projeteur peut être grand lorsqu'il doit choisir une valeur dans la fourchette pour sa décision. Quelle valeur choisir, comment la décision de dimensionnement d'un projet doit-elle prendre en compte l'incertitude des données?

En corollaire à la question précédente se pose le problème de la valeur informative des données. Sont-elles suffisantes pour que le projeteur obtienne une décision correcte? Certes les problèmes de protection sont à une urgence telle qu'il serait vain d'attendre les observations hydrométriques de plusieurs années complémentaires par exemple, mais peut-être faut-il envisager la manière la plus efficace de collecter les données nécessaires à la prise des décisions? En fait les informations disponibles sont de natures diverses, quantitatives ou même qualitatives, leur prise en compte a souvent donné naissance à des méthodes présentées comme concurrentes (analyse statistique des débits maximaux annuels, méthode du gradex, méthodes déterministes de transfert précipitation-écoulement appliquées à des "précipitations maximales probables", etc...). Mais dans un cadre décisionnel, ces méthodes doivent-elles réellement être concurrentes et ne faut-il pas les reconcilier pour aboutir à la meilleure décision?

Il est remarquable que toutes les réponses aux problèmes et questions précédents peuvent être reliées dans le cadre de la Théorie de la Décision Statistique et de l'application des méthodes dites bayesiennes dont les travaux rapportés dans la suite sont des illustrations. Cette théorie n'est pas neuve, l'exposé le plus complet de ses principes a été donné par Savage en 1954 [8] et les outils pratiques d'application par Raiffa et Schlaifer en 1961 [7]. Dans le domaine de l'hydrologie les premières applications datent de 1967 [1] et 1972 [3].

Les probabilités de dépassement des crues extrêmes

Le point de départ de la théorie concerne l'interprétation concrète d'une notion théorique de probabilité 
telle que $p$ : probabilité de dépassement d'un débit maximal annuel $x$ donné. Dans le sens traditionnel une probabilité est conçue comme la limite d'une fréquence d'événements dans une longue suite d'épreuves. Les hydrologues utilisent la notion de durée de retour, inverse de $p$, intervalle de temps moyen séparant deux occurrences successives d'un débit maximal supérieur à $x$, ce qui sous-entend une interprétation "fréquentiste". On a souvent dit que cette interprétation était difficilement concevable pour des crues millénalles ou même centenalles par exemple. Cela n'empêche pas les hydrologues d'utiliser la notion de crue millénalle de façon opérationnelle pour des problèmes de décision face au risque. Mais cette utilisation et les calculs probabilistes qui en découlent sont-ils justifiés si l'interprétation fréquentiste n'est pas possible ? Savage [8] a montré qu'il pouvait exister une autre interprétation concrète de valeur opérationnelle : la probabilité subjective, introduite par une certaine formalisation du comportement d'un décideur face à l'incertitude. Depuis Savage, plusieurs présentations de la théorie ont été faites notamment par Morlat [5] dans une communication récente relative au traitement des événements rares. Nous rappelons, en annexe, l'exposition plus élémentaire de PrattRaiffa et Schlaifer [6].

Les principes de cette théorie permettent de démontrer l'existence des probabilités subjectives $P\left(E_{i}\right)$ affectées aux états de la nature qui intéressent le décideur. Concrètement l'estimation séparée des $P\left(E_{i}\right)$ pour chaque état de la nature séparé de son environnement pourrait être une tâche très lourde ; cela n'est pas nécessaire. Le décideur peut apprécier directement un petit nombre de probabilités $P\left(E_{i}\right)$ et extrapoler ou interpoler les autres par une formule.

Considérons par exemple la loi de probabilité du débit maximal annuel $X_{M}$ d'un bassin donné :

$$
\text { Prob }\left[X_{M} \leqslant x\right]=F(x ; \mu, \rho)
$$

On adopte généralement une forme de loi qui dépend de paramètres, généralement deux comme $\mu$ et $\rho$. Le décideur peut considérer les différentes valeurs de $x$ possibles comme les états de la nature : cette interprétation fonde alors les probabilités de crues maximales annuelles en tant que probabilités subjectives mais une conception plus large est possible, celle que nous adopterons dans la suite:

- pour le décideur, un état de la nature est une loi $F$ entièrement spécifiée, c'est-à-dire en fait un couple spécifié de valeurs des paramètres $(\mu, \rho)$. Dans le cadre de la théorie de Savage $(\mu, \rho)$ constitue alors un couple de variables aléatoires dont il peut évaluer les probabilités en calant une formule sur les informations qu'il possède tout comme il ajuste une loi de probabilités empirique sur les observations de débit pour choisir la loi $F$.

\section{Un modèle probabiliste de débit maximal annuel}

Les hydrologues connaissent bien les méthodes statis. tiques classiques d'analyses des débits maximaux annuels qui utilisent, pour estimer la loi $F$, des échantillons d'observations de débits maximaux annuels et qui ont pour inconvénients de ne pas prendre en compte des débits de crues importantes dans la mesure où leur débit est inférieur au débit maximal annuel. Nous envisageons une méthode plus informative (cf. [4]) qui a, en outre, l'avantage d'une mise en œuvre plus simple de la théorie de la Décision :

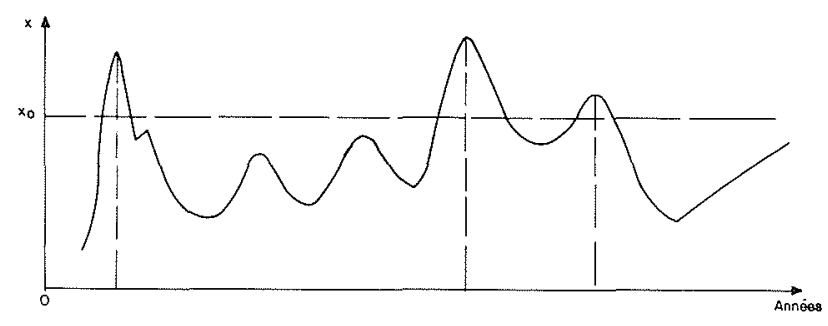

A partir d'un seuil $x_{0}$ convenablement choisi, on sélectionne les pointes de crue de débit $x$ (débit moyen journalier maximal ou maximum instantané) supérieur au seuil $x_{0}$. L'analyse porte à la fois sur tous les débits $x$ et sur les nombres annuels de crues ainsi sélectionnées.

Moyennant l'hypothèse d'indépendance entre les débits des crues sélectionnées (hypothèse vérifiée généralement lorsque le seuil $x_{0}$ n'est pas trop bas) et si :

- $K\left(x, x_{0}\right)$ est la loi de probabilité du débit d'une crue sélectionnée :

$$
K\left(x, x_{0}\right)=\operatorname{Prob}\left[X \leqslant x / x_{0}\right]
$$

$P_{n}$ est la probabilité du nombre annuel de crues sélectionnées. Alors la loi du débit maximal annuel s'écrit:

$$
\operatorname{Prob}\left[X_{M} \leqslant x\right]=\sum_{n=0}^{\infty} P_{n} K^{n}\left(x, x_{0}\right)
$$

Un modèle paramétrique possible et souvent rencontré spécifie :

- la loi de Poisson $P_{n}$ :

$$
P_{n}=e^{-\mu} \frac{\mu^{n}}{n !}
$$

- la loi exponentielle pour une fonction $\psi\left(x, x_{0}\right) \mathrm{du}$ débit :

$$
K=1-e^{-\rho \psi\left(x, x_{0}\right)}
$$

Avec ces hypothèses, on obtient :

$$
F(x, \mu, \rho)=\operatorname{Prob}\left[X_{m} \leqslant x\right]=e^{-\mu e^{-\rho \psi\left(x, x_{0}\right)}}(4)
$$

Ce modèle est compatible avec les lois de probabilités classiques des valeurs extrêmes (lois de Gumbel et Frechet) que l'on retrouve respectivement pour $\psi\left(x, x_{0}\right)=x-x_{0}$ et $\psi\left(x, x_{0}\right)=\log x-\log x_{0}$ (On trouvera des illustrations de ce modèle dans [2] et [4]).

La mise en œuvre pratique d'un tel modèle s'avère très simple; considérons $r$ années successives où on a observé au total $N$ crues $x_{i}$ supérieures au seuil telles que :

$$
\sum_{i=i}^{n} \psi\left(x_{i}, x_{0}\right)=S
$$

on peut estimer les paramètres de la façon suivante :

$$
\bar{\mu}=\frac{N}{r}
$$




$$
\bar{\rho}=\frac{1}{S} \text { avec } \bar{S}=\frac{S}{N}
$$

Cette méthode d'estimation est la meilleure possible ; elle utilise de façon exhaustive toute l'information contenue dans les observations de débit sur les $r$ années relativement aux paramètres $\mu$ et $\rho$. C'est toutefois la méthode d'estimation classique indépendante de toutes idées a priori sur les paramètres. La théorie de Savage permet une approche différente du problème d'estimation.

\section{Le théorème de Bayes}

Le caractère informatif des statistiques $N$ et $S$ utilisées dans la méthode d'estimation précédente est défini par les lois de probabilité de ces statistiques pour $\mu$ et $\rho$ fixés, on trouve facilement que :

$-N$ est une variable de Poisson

$$
P_{N}(\mu)=e^{-r \mu} \frac{(r \mu)^{N}}{N !}
$$

- si $N$ est supposée fixée, $S$ distribuée selon une loi gamma de densité :

$$
\frac{\rho^{N}}{\Gamma(N)} e^{-\rho S} S^{N-1}
$$

Pour revenir à la théorie de la probabilité subjective, on considère les paramètres $\mu$ et $\rho$ comme des variables aléatoires ; on suppose que les informations a priori (avant prise en compte des $r$ années d'observations) du décideur peuvent être résumées par des distributions gamma sur $\mu$ et $\rho$, soit leurs densités :

$$
\begin{aligned}
& -g_{1}^{\circ}(\mu)=\frac{1}{\mu_{0} \Gamma\left(\gamma_{0}\right)} e^{-\frac{\mu}{\mu_{0}}}\left(\frac{\mu}{\mu_{0}}\right)^{\gamma_{0}-1} \\
& -g_{2}^{\circ}(\rho)=\frac{1}{\rho_{0} \Gamma\left(\delta_{0}\right)} e^{-\frac{\rho}{\rho_{0}}}\left(\frac{\rho}{\rho_{0}}\right)^{\delta_{0}-1}
\end{aligned}
$$

Ces formes ont été choisies pour des raisons de commodité qui apparaîtront ultérieurement. Toutefois leur comportement est assez souple pour représenter les incertitudes sur $\mu$ et $\rho$ les plus diverses.

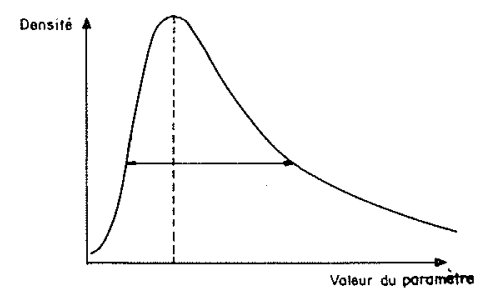

Pratiquement on peut estimer les probabilités de quelques valeurs de $\mu$ et $\rho$ et interpoler par un choix convenable des valeurs de $\mu_{0}, \rho_{0}, \gamma_{0}$ et $\delta_{0}$. On peut également choisir a priori les moyennes et variances a priori et déterminer les paramètres correspondants. Nous y reviendrons plus loin ; en attendant les moments des distributions sont utiles à considérer :

$$
E_{0}(\mu)=\gamma_{0} \mu_{0} \quad \operatorname{Var}_{0}(\mu)=\gamma_{0} \mu_{0}^{2}
$$

d'où

$$
\mu_{0}=\frac{\operatorname{Var}_{0}(\mu)}{E_{0}(\mu)} \quad \gamma_{0}=\frac{E_{0}^{2}(\mu)}{\operatorname{Var}_{0}(\mu)}=\frac{1}{C V_{0}^{2}(\mu)}
$$

de même pour $\rho$ :

$$
E_{0}(\rho)=\delta_{0} \rho_{0} \quad \operatorname{Var}_{0}(\rho)=\delta_{0} \rho_{0}^{2}
$$

d'où

$$
\rho_{0}=\frac{\operatorname{Var}_{0}(\rho)}{E_{0}(\rho)} \quad \delta_{0}=\frac{E_{0}^{2}(\rho)}{\operatorname{Var}_{0}(\rho)}=\frac{1}{C V_{0}^{2}(\rho)}
$$

Les probabilités a priori sur $\mu$ et $\rho$ doivent être modifiées par les observations des $r$ années de débits ; le théorème de Bayes explicite la relation entre probabilités a posteriori ainsi modifiées et probabilités a priori. Prenons pour exemple le paramètre $\mu$ pour lequel :

$g^{\circ}(\mu)$ est la densité a priori

$P_{N}(\mu)$ est la probabilité d'observer $N$ pour $\mu$ fixé.

Le théorème de Bayes donne l'expression de la densité de $\mu$ a posteriori $g^{N}(\mu)$ :

$$
g^{N}(\mu)=\text { constante } \times P_{N}(\mu) \times g^{\circ}(\mu)
$$

Avec les hypothèses du modèle, on obtient une nouvelle distribution gamma de la forme (9) mais où $\mu_{0}$ est remplacé par $\frac{\mu_{0}}{r \mu_{0}+1}$ et $\gamma_{0}$ par $\gamma_{0}+N$. On montrerait de la même façon que la distribution a posteriori de $\rho$ est encore une distribution gamma où $\rho_{0}$ est remplacé $\operatorname{par} \frac{\rho_{0}}{S \rho_{0}+1}$ et $\delta_{0}$ par $\delta_{0}+N$. L'utilisation du théorème de Bayes explique le nom de méthodes bayesiennes donné à ces techniques.

La donnée de la distribution a posteriori peut permettre la résolution du problème d'estimation. Prenons le cas de $\mu$ :

- on peut estimer $\mu$ par une caractéristique de tendance centrale de la distribution, par exemple l'espérance mathématique :

$$
E(\mu)=\frac{\left(\gamma_{0}+N\right) \mu_{0}}{r \mu_{0}+1}
$$

- on peut calculer un intervalle de confiance bayesien de seuil de confiance $\alpha$, en déterminant deux limites $\mu_{1}$ et $\mu_{2}$ telles que :

$$
\operatorname{Prob}^{N}\left[\mu_{1} \leqslant \mu \leqslant \mu_{2}\right]=\alpha
$$

L'exposant $N$ précise ici que le calcul est à faire avec la loi a posteriori de $\mu$.

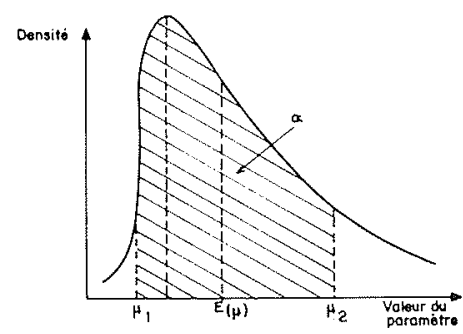

\section{Remarques :}

On peut voir facilement que si la taille de l'échan. 
tillon augmente, $E(\mu)$ tend vers $\mu=N / r$ (estimation classique) qui est indépendant des paramètres a priori $\mu_{0}$ et $\gamma_{0}$; on peut résumer ce résultat en disant que le poids des idées a priori diminue et tend vers zéro à mesure que l'information augmente.

On peut représenter l'absence de toute information a priori dans ce schéma en posant $\gamma_{0}=0$ et en faisant tendre $\mu_{0}$ vers l'infini.

\section{Estimation bayesienne d'un quantile}

Des résultats analogues peuvent ètre trouvés pour $\rho$ puisque la même forme de loi gamma s'applique. Toutefois il est peut être intéressant ici d'étudier directement l'estimation d'un quantile, c'est-à-dire un débit maximal annuel $x_{p}$ dont la probabilité de dépassement, calculée avec notre modèle, est $p$, soit :

$$
e^{-\mu e^{-\rho \psi\left(x_{p}, x_{0}\right)}}=1-p
$$

ce qui donne :

$$
\psi\left(x_{p}, x_{0}\right)=\frac{\log \mu+y_{p}}{\rho}
$$

avec

$$
y_{P}=\log [-\log (1-p)]
$$

Compte tenu de cette formule, il faudrait en toute rigueur combiner les distributions a posteriori de $\mu$ et $\rho$ pour obtenir la loi de probabilité a posteriori de $\psi$.

Une approximation est cependant possible dans la plupart des applications :

Lorsqu'il s'agit d'estimer des crues millénalles ou centenalles $\left(P=\frac{1}{1000}\right.$ ou $\left.\frac{1}{100}\right) y_{p}$ est grand devant la valeur moyenne de $\log \mu$ et ses écarts aléatoires. On peut donc s'affranchir des incertitudes sur $\mu$ en remplaçant $\mu$ par son espérance a posteriori ; en fait le paramètre le plus sensible est $\rho$ :

$$
\psi\left(x_{p}, x_{0}\right) \simeq \frac{\log E(\mu)+y_{p}}{\rho}
$$

soit

$$
\psi\left(x_{p}, x_{0}\right) \simeq \frac{\frac{\log \mu_{0}\left(\gamma_{0}+N\right)}{r \mu_{0}+1}+y_{p}}{\rho}
$$

Un intervalle de confiance sur $\psi$ peut alors être déduit d'un intervalle de confiance sur $\rho$ calculé par une formule analogue à (17) puisque $\rho$ est, comme $\mu$, distribué selon une loi gamma, de paramètres différents toutefois.

\section{Exemple d'application}

Nous prenons ici un exemple relativement défavorable du point de vue des incertitudes sur le paramètre $\mu$. Il s'agit de l'estimation de la crue millénalle de la Sioule (affluent de l'Allier) à la station de Pont du Bouchet en utilisant les 44 années d'observations entre 1919 et 1962 et en choisissant un seuil $x_{0}$ assez élevé égal à $120 \mathrm{~m}^{3} / \mathrm{s}$ (en débit moyen journalier). La fonction $\psi\left(x, x_{0}\right)=x-x_{0}$ a été adoptée ici :

$$
\begin{gathered}
\text { On a donc obtenu : } \\
r=44, \quad N=25, \quad S=\sum_{i=1}^{25}\left(x_{i}-x_{0}\right)=1672\left(\mathrm{~m}^{3} / \mathrm{s}\right)
\end{gathered}
$$

Les intervalles de confiance pour $\alpha=95 \%$ sont donnés pour des distributions a priori non informatives $\left(\mu_{0}=\rho_{0}=\infty ; \gamma_{0}=\delta_{0}=0\right)$ :

$0,363 \leqslant \mu \leqslant 0,806 \quad$ valeur moyenne $E(\mu)=0,568$

$0,0096 \leqslant \rho \leqslant 0,0212$ valeur moyenne $E(\rho)=0,0150$

Dans le cas d'une crue millénalle, $y_{p}=6,91$; l'importance relative des incertitudes sur $\mu$ et $\rho$ est illustrée par le fait suivant : les limites extrêmes des intervalles précédents donnent des écarts sur $x_{p}-x_{0}$ de $13 \%$ pour ce qui concerne $\mu$ et $122 \%$ pour ce qui concerne $\rho$. L'approximation (17) semble donc justifiée ; elle fournit l'intervalle de confiance approché pour $x_{p}$ dans le cas d'un seuil de confiance de $95 \%$

$$
419 \mathrm{~m}^{3} / \mathrm{s} \leqslant x_{p} \leqslant 661 \mathrm{~m}^{3} / \mathrm{s}
$$

\section{Prise en compte des informations a priori}

Les méthodes bayesiennes sont caractérisées par l'intervention des probabilités a priori. Leurs estimations pourront sembler assez arbitraires à certains pour que des doutes soient jetés à l'encontre de ces techniques. Dans le cadre de la théorie, ces probabilités sont appelées subjectives mais cela ne veut pas dire arbitraires; certes elles concernent un individu particulier confronté à l'incertitude avec ses informations propres. Deux individus peuvent ainsi aboutir à estimer différemment les probabilités d'un même évènement ; toutefois la prise en compte des informations obéit à des règles de cohérence indiquées par les postulats de la théorie dont une des conséquences essentielles est l'utilisation du théorème de Bayes.

En matière d'études de crues les informations sont diverses : données géomorphologiques, données météorologiques, notamment pluviométriques, données hydrométriques ; leur qualité est variable : observation systématique de réseaux, relevés épisodiques, historiques plus ou moins précis (comme les laisses de crues) dont le contenu informatif n'est pas négligeable. La disponibilité de ces informations diverses a donné naissance à des méthodes de calcul présentées souvent comme concurrentes. L'apport, peut-être le plus important, des méthodes bayesiennes est de permettre une synthèse cohérente et rationnelle de toutes ces informations en vue de résoudre efficacement un problème de décision. Pour cela il importe de caler les probabilités a priori sur le type d'information que l'on veut prendre en compte avant traitement des informations hydrométriques systématiques par exemple. Le fait de considérer des formes mathématiques particulières comme les distributions a priori gamma par les paramètres $\mu$ et $\rho$ permet de faciliter ce calage. On a noté que le coefficient de variation de ces paramètres : Ecarttype/Espérance, qui peut être assimilé à une erreur relative est directement relié aux caractéristiques a priori $\gamma_{0}$ et $\delta_{0}$ (formules (13), (14)). Par ailleurs une 
fourchette $\left[X_{M 1}, X_{M 2}\right]$ sur les débits maximaux annuels, obtenue par le traitement des données géomorphologiques ou pluviométriques peut fournir des ordres de grandeur de $\mu_{0}$ et $\rho_{0}$. Une telle procédure a été utilisée dans [1]. Bernier et Miquel [2] ont montré comment les données épisodiques historiques pouvaient être intégrées avec une telle méthodologie.

\section{Le gradex des précipitations}

On connaît le succès rencontré par la méthode d'estimation des probabilités de crues basée sur le gradex des précipitations, développée par MM. Duband et Guillot (voir [4]). Rappelons que la distribution des précipitations journalières $y$ est représentée dans cette méthode par une loi exponentielle :

$$
\text { Prob }[Y \geqslant y]=e^{\frac{-y-y_{0}}{a}} \text { avec } y \geqslant y_{0}
$$

où $a$ est le paramètre nommé le gradex des pluies. Cette formule est cohérente avec notre modèle tenant compte d'une distribution exponentielle des crues supérieures à un seuil (pour $\psi=x-x_{0}$ ) et nous l'écrirons :

$$
\text { Prob }[Y \geqslant y]=e^{-\rho_{a}\left(y-y_{0}\right)} \text { avec } \rho_{a}=\frac{1}{a}
$$

L'hypothèse fondamentale de la méthode du gradex est l'identité du gradex des précipitations et du gradex des débits maximaux à l'échelle journalière ; soit :

où

$$
\begin{gathered}
\rho=\rho_{a} \\
\rho=\lambda \rho_{a}
\end{gathered}
$$

s'il s'agit du débit de crue maximal instantané [4], dans ce cas $\lambda$ est supposé parfaitement connu.

Cette hypothèse a été souvent soumise à critique et il n'est pas sans intérêt d'analyser les incertitudes (au sens bayesien) sur le coefficient de passage $\lambda$ entre les deux gradex. Du fait de la cohérence théorique, soulignée plus haut entre les deux modèles, on peut représenter l'incertitude sur $\rho_{a}$ par une distribution gamma; dans le cadre de cette loi, le carré des coefficients de variation $\left(C V^{2}\right)$ de $\rho$ et de $\rho_{a}$ peut s'interprêter (cf. formule (14)) comme l'inverse de nombres d'observations sur les débits $N$ ou précipitations $M$ nécessaires pour obtenir la précision déterminée par ce coefficient de variation sur les gradex ; on obtient :

$$
N=\frac{M}{1+(M+1) C V^{2}(\lambda)}
$$

Cette formule donne la quantité d'informations sur $\rho$ mesurée par $N$, apportée par $M$ observations de précipitations compte tenu de l'incertitude éventuelle sur $\lambda$ mesurée par son coefficient de variation $C V(\lambda)$.

L'information est certes maximale $(N=M)$ si la certitude sur $\lambda$ est absolue $(C V=0)$ mais elle décrồt lorsque l'incertitude augmente. Pour un $C V(\lambda)$ fixé, on observera que $N$ augmente avec $M$ mais que, de toutes façons, elle atteint asymptotiquement une borne supérieure égale à : $1 / C V^{2}(\lambda)$ qui donne la limite du gain d'information sur l'estimation des débits de crue apportée par le gradex des précipitations pour autant qu'une incertitude sur le passage du gradex des précipitations au gradex des débits existe.

\section{Analyse plus complète du problème décisionnel}

Soit une structure destinée à la protection contre les crues dont le dimensionnement est défini par le choix d'un débit critique $x_{c}$, appelé débit de crue de projet. Dans le cadre de la Théorie de la Décision Statistique, il importe d'envisager les conséquences du choix de $x_{c}$, à savoir :

- un investissement $I\left(x_{c}\right)$

- des dommages que la structure construite ne permettra pas d'éviter.

On notera que, au sens de la théorie, ces dommages doivent comprendre tout ce qui peut amener le décideur à modifier son choix ; il s'agit donc de conséquences dommageables dont certaines peuvent être exprimées en termes monétaires, alors que d'autres ne sont pas immédiatement quantifiables. Toutefois on remarquera, en se rapportant aux postulats de la théorie (voir annexe), que ceux-ci fondent non seulement la notion de probabilité mais aussi la notion d'utilité (négative dans le cas présent) qui quantifie les conséquences des décisions. Aussi peut-on exprimer les dommages par une fonction numérique $D\left(x, x_{c}\right)$, que nous supposons dépendre à la fois de la variable de décision $x_{c}$ et de la valeur $x \mathrm{du}$ débit maximal $X$ de la crue qui provoque ces dommages (on supposera $D=0$ si $x<x_{c}$ ). Du point de vue de l'utilité, on peut exprimer $D$ avec les mêmes mesures unités (monétaires) que l'investissement $I\left(x_{c}\right)$. La mise en œuvre concrète de la théorie pose certes certains problèmes pour l'estimation de l'utilité ; il faut en retenir l'idée que si une évaluation monétaire est tentée, elle doit concerner le maximum de conséquences quantifiables. L'interprétation en terme d'utilité pour le décideur est aussi à prendre en compte s'il s'agit d'extrapoler la fonction de dommage au delà des grandes crues pour lesquelles l'information économique a pu être collectée.

Compte tenu des aléas sur les débits, facteurs de dommages, on aura à considérer une fonction $H\left(x_{c}\right)$ : somme de l'investissemient et de l'espérance mathématique des dommages convenablement actualisés pour tenir compte de leur échelonnement dans le temps. Avec les hypothèses du modèle de crue que nous avons adoptées, on obtient :

$$
\begin{aligned}
& H\left(x_{c} ; \mu, \rho\right)=I\left(x_{c}\right) \\
& \quad+k \cdot \mu \int_{x_{c}}^{\infty} D\left(x, x_{c}\right) \rho e^{-\rho \psi\left(x, x_{c}\right)} d x^{(*)}(23)
\end{aligned}
$$

Considérons, en premier lieu, le cas où les paramètres $\mu$ et $\rho$ sont parfaitement connus. La méthode de choix de la décision revient donc à minimiser l'utilité négative exprimée par $H\left(x_{c}\right)$.

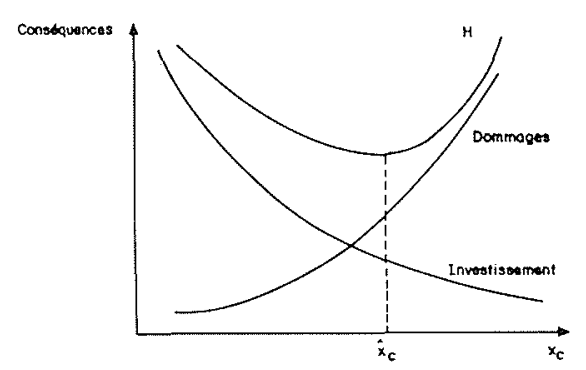


Un résultat important doit être noté : si la fonction de dommages est linéaire :

$$
D=\beta\left(x-x_{c}\right)
$$

On obtient une solution optimale :

$$
I-F\left(\hat{x}_{c} ; \mu, \rho\right)=p=\frac{d I / d c}{k \beta}
$$

Le débit $\hat{x}_{c}$ de la crue de projet optimal est ainsi égal au débit maximal annuel dont la probabilité de dépassement s'exprime comme le rapport du coût d'investissement marginal au coût marginal actualisé $k \beta$. Ce résultat important est approximativement valable pour autant que le dommage $D\left(x, x_{c}\right)$ soit approximativement linéaire dans une plage de débit $x$ entourant l'optimum $\hat{x}_{c}$. Si le coût marginal d'investissement est également constant, on retrouve les errements classiques où la décision est basée sur le choix d'une probabilité $p$ forfaitaire, mais on voit ici que cette probabilité a une interprétation précise en termes de conséquences de la décision.

Si $\mu$ et $\rho$ ne sont pas parfaitement connus, cas habituel, on doit prendre l'espérance de $H$ pondérée par les probabilités affectées à ces paramètres, soit :

$H^{*}\left(x_{c}\right)=I\left(x_{c}\right)+k E(\mu)$.

$$
\int_{x_{c}}^{\infty} D\left(x, x_{c}\right) E\left[\rho \psi^{\prime} e^{-\rho \psi}\right] d x
$$

$\psi^{\prime}$ est la dérivée de la fonction $\psi$ par rapport à $x$. Compte tenu de la distribution gamma représentant les incertitudes sur $\rho$, on obtient:

$$
\begin{aligned}
& H^{*}\left(x_{c}\right)=I\left(x_{c}\right)+k E(\mu) . \\
& E(\rho) \int_{x_{c}}^{\infty} \frac{D\left(x, x_{c}\right) \cdot \psi^{\prime}}{\left[\rho_{0} \psi\left(x, x_{c}\right)+1\right]^{\delta_{0}+1}} d x
\end{aligned}
$$

Dans cette formule $\rho_{0}$ et $\delta_{0}$ prennent les valeurs a posteriori tenant compte de toutes les informations disponibles. On observe que l'incertitude sur $\mu$ n'intervient que par son espérance mathématique alors que l'incertitude sur $\rho$ est représentée de façon plus complexe par les paramètres caractérisant sa distribution, ce qui illustre encore le caractère dissymétrique des deux paramètres du modèle.

Revenons au cas où les coûts marginaux sont constants de telle sorte que leur rapport s'exprime par la probabilité de dépassement de la crue de projet dans le cas de connaissance parfaite des paramètres. Le rapport inverse $\frac{k \beta}{d l / d c}$ s'exprime alors comme une durée de retour $T$ inverse de $p$.

La valeur de $\stackrel{\hat{x}}{\text { minimisant }} H^{*}\left(x_{c}\right)$ est donnée par :

$$
\psi\left(\hat{\hat{x}}_{c}, x_{0}\right) \simeq \frac{\log T E(\mu)}{E(\rho)}+\frac{1}{2} \frac{\operatorname{Var}(\rho)}{E^{3}(\rho)}\left[\log (T E(\mu)]^{2}\right.
$$

* $k$ est un coefficient d'actualisation qui peut tenir compte éventuellement d'une évolution des dommages au cours du temps du fait notamment de l'évolution économique des zones concernées par ces dommages.
L'optimum sur $\psi$ est donc la somme de deux termes:

- le premier terme correspond à la valeur optimale que l'on obtiendrait si les paramètres étaient parfaitement connus et égaux à leurs expérances mathématiques ;

- le terme complémentaire est proportionnel à Var $(\rho)$ et représente le sur-dimensionnement nécessité par l'incertitude sur le paramètre $\rho$.

De façon parallèle on pourrait montrer que :

$$
H^{*}\left(x_{c}\right)>H\left(x_{c} ; E(\mu), E(\rho)\right)
$$

L'écart $H^{*}-H$ traduit en fait le prix que l'on doit payer à l'incertitude sur les paramètres, essentiellement $\rho$ d'ailleurs. La comparaison de $H^{*}$ à l'optimum avec ce que l'on obtiendrait si les paramètres étaient parfaitement connus est à la base d'une méthode d'évaluation du coût de l'incertitude et de la valeur "économique" de l'information qui réduit cette incertitude.

\section{Remarques importantes}

L'analyse des incertitudes intervenant dans un problème de protection contre les crues, telle qu'elle a été évoquée ci-dessus est loin d'être complète. On a fixé notre attention sur les paramètres $\mu$ et $\rho$ du modèle mais ce modèle lui-même est incertain : nos connaissances et informations nous permettent-elles de choisir à coup sûr la fonction $\psi\left(x, x_{0}\right)$ notamment ? Les hydrologues connaissent bien le problème difficile du choix du support d'extrapolation des lois de probabilités des crues et les décisions entre ajustement de Gumbel, de Frechet, etc...

Les incertitudes sur la fonction de dommages sont peut être plus considérables encore si tant est que l'on puisse les quantifier complètement d'ailleurs. Toutefois la formulation de la théorie de la décision permet la prise en compte de ces incertitudes comme celles d'origine hydrologique.

Un résultat peut ètre intéressant à noter : dans le cas de coût marginal de dommage $\beta$ constant (formule (24)) la fonction critère $H^{*}$ est uniquement fonction de $E(\beta)$, espérance mathématique de la distribution a priori ou a posteriori de ce coût marginal.

Ainsi au moins un certain nombre des incertitudes peuvent être prises en compte par une analyse bayesienne; mais toute l'information aura nécessairement des limites, c'est une des raisons pour lesquelles des optimisations de décision du type précédent dans le cadre d'une analyse partielle ne fournissent pas nécessairement la solution finale. Toutefois une analyse décisionnelle plus ou moins complète est utile car elle permet une classification de diverses incertitudes et une mobilisation cohérente de toutes les informations disponibles pour les réduire ; elle permet aussi la mise en évidence de la sensibilité de la précision de certains paramètres et du choix de certaines hypothèses plus ou moins masquées dans les méthodes courantes.

\section{Le barrage des Angles}

Les principes méthodologiques de la Théorie Statistique de la Décision ont fait l'objet de tentatives d'appli- 


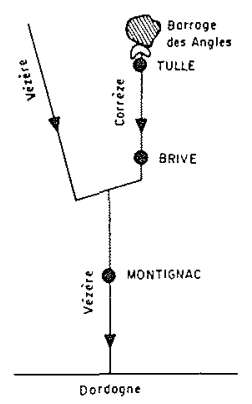

cation à l'étude du barrage des Angles, un projet situé sur la Corrèze à l'amont de Tulle (voir schéma ci-joint), destiné à écrêter les crues dommageables à l'aval sur la Corrèze et la Vézère.

Un tel cas d'application est plus complexe que le modèle décrit précédemment :

- il y a bien une seule variable de dimensionnement, ici le volume $V$ du réservoir des Angles, mais les effets de l'écrêtement se font sentir sur tout le cours aval de la Corrèze et de la Vézère après son confluent avec la Corrèze. Il n'est pas douteux qu'il y, a là de nombreuses incertitudes sur la propagation des crues naturelles ou écrêtées et donc sur l'effet supposé du réservoir ;

- les conséquences d'une crue sur la Corrèze concernent tout l'aval ; nous avons schématisé celle-ci en intégrant ces conséquences au niveau de trois points critiques : Tulle, Brive et Montignac ; nous avions donc :

- trois fonctions de dommages estimées par des fonctions linéaires,

- trois lois de probabilités des débits extrêmes, indexées par 6 paramètres au total (un couple $\mu, \rho$ pour chacun des points critiques).

Seule l'incertitude sur les données hydrologiques a été analyée dans cette étude.

$\mathrm{La}$ figure jointe illustre la variation de la somme : investissements + espérance des dommages évités par la construction du barrage en fonction du volume du réservoir. (Ces dommages sont ici actualisés au taux de $10 \%$ ).

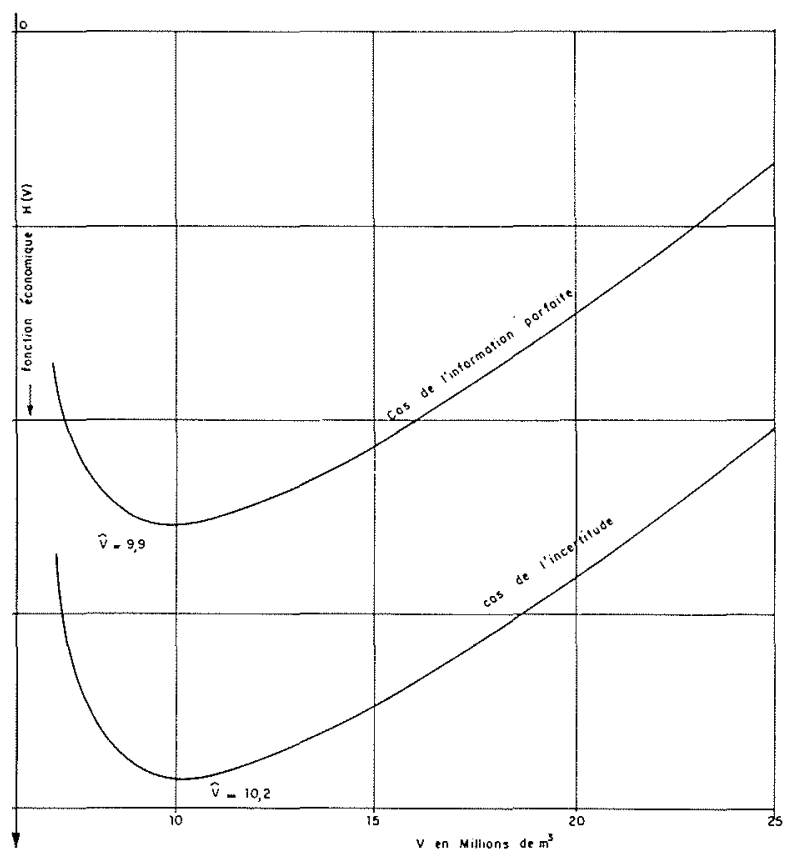

Barrage des Angles - Dommages évités et investissements $H(v)$ en fonction du volume du réservoir.

\section{Bibliographie}

[1]BERNIER J. - Les méthodes bayesiennes en hydrologie statistique. $1^{\text {er }}$ Symposium International sur l'Hydrologie Mathématique. Fort Collins, 1967.

[2] BERNIER J., MIQUEL J. - Exemple d'application de la théorie de la décision statistique au dimensionnement d'ouvrages hydrauliques : prise en compte de l'information hétérogène. $17^{e}$ Congrès de l'A.I.R.H., Baden-Baden, 1977.

[3] DAVIS D.R., KISIEL C.C., DUCKSTEIN L. - Bayesian Decision Theory Applied to Design in Hydrology. Water Resources Research, 1972.

[4] DUFFAUT J. et al. - Détermination des crues de projet : Groupe de travail du Comité des Grands Barrages. $11^{e}$ Congrès des Grands Barrages, Madrid, 1973.

[5] MORLAT G. - Sur la théorie de la Décision appliquée aux évènements rares. Réunion $O C D E$ sur les problèmes d'évènements rares dans l'analyse de fiabilité des centrales nucléaires, Ispra, Juin 1976

[6]PRATT - RAIFFA - SCHLAIFER. - The Foundation of Decision under Uncertainty. An Elementary Exposition. American Statistical Association Journal, Tome 59, $\mathrm{n}^{\circ} 30$, Juin 1964.

[7]RAIFFA - SCHLAIFFER. - Applied Statistical Decision Theory. Harvard University Press, 1961.

[8] SAVAGE L.J. - Foundations of Statistics. Wiley, New York, 1954.

\section{ANNEXE}

Exposé des principes de la théorie statistique de la décision d'après Pratt - Raiffa - Schlaifer [6]

\section{La base canonique}

Considérons une personne désirant analyser logiquement sa procédure de décision pour un problème particulier. Il commence par se définir l'ensemble des décisions ou actes entre lesquels il doit choisir et les conséquences $H_{i j}$ de chacun de ces actes $a_{i}$ associées aux évènements $E_{j}$ mutuellement exclusifs qui peuvent survenir et modifier ainsi les conséquences.

\begin{tabular}{l|llll} 
Actes & \multicolumn{5}{|c}{ Evènements } \\
\hline & $E_{1}$ & $E_{2}$ & $E_{j}$ & $E_{r}$ \\
$a_{1}$ & $I_{11}$ & $H_{12}$ & $H_{2 j}$ & $H_{i r}$ \\
$a_{2}$ & & & & \\
$a_{i}$ & $H_{i 1}$ & $H_{i 2}$ & $H_{i j}$ & $H_{i r}$ \\
$a_{m}$ & $H_{m 1}$ & $H_{m 2}$ & $H_{m j}$ & $H_{m r}$
\end{tabular}

Pour comparer les actes possibles entre eux, on suppose que le décideur peut (comme un expérimentateur se réfère à un mètre étalon pour mesurer des longueurs) se référer à des loteries hypothétiques, dites canoniques ayant les propriétés suivantes :

\section{Première hypothèse :}

1/ Le décideur peut imaginer une expérience (dite canonique) dont les résultats sont "également vraisemblables" dans le sens où le décideur serait indifférent entre deux loteries dont l'une lui donne un certain prix si un résultat particulier de l'expérience canonique sur- 
vient et l'autre lui donne le même prix si un autre résultat particulier survient.

2/ Etant donné deux loteries canoniques dont l'une lui donne un prix si un quelconque parmi $n_{1}$ résultats particuliers de l'expérience canonique survient et l'autre lui donne le même prix si un quelconque parmi $n_{2}$ résultats particuliers survient, alors il préfèrera la première loterie si $n_{1}>n_{2}$.

Si une expérience canonique a $K$ résultats possibles, on peut donc dire que la "probabilité canonique" d'un résultat quelconque est, pour le décideur, égale à $1 / K$. La probabilité canonique de l'un quelconque des $k$ résultats également vraisemblables est alors égale à $k / K$.

\section{Relations d'ordre sur les préférences et jugements.}

\section{Deuxieme hypothèse.}

Le décideur peut se référer à des conséquences standard $\vec{H}$ et $\underline{H}$ telles que $\bar{H}$ est au moins aussi bonne que l'une quelconque des conséquences de n'importe quel acte et $\bar{H}$ est au moins aussi mauvaise que l'une quelconque des conséquences de n'importe quel acte ; alors :

- pour une conséquence quelconque $H$, il mesurera sa préférence en spécifiant un nombre $\pi(H)$ tel que : il serait indifférent entre une loterie lui garantissant $H$ de façon certaine et une loterie donnant une probabilité canonique $\pi(H)$ à $\bar{H}$ et $1-\pi(H)$ à $\underline{H}$; - pour un événement particulier $E_{0}$, il mesurera son jugement en spécifiant un nombre $P\left(E_{0}\right)$ tel que : il serait indifférent entre une loterie dont les conséquences sont $\bar{H}$ si $E_{0}$ survient et $\underline{H}$ si $E_{0}$ ne survient pas et une loterie donnant une probabilité canonique $P\left(E_{0}\right)$ à $\bar{H}$ et $1-P\left(E_{0}\right)$ à $\underline{H}$.

Pour appliquer les principes précédents le décideur n'a pas besoin de faire son évaluation $P\left(E_{0}\right)$ pour tous les événements possibles de son environnement; dans le cas où ces événements sont associés à des valeurs numériques (débits de crues ou valeurs des paramètres $\mu$ et $\rho$ par exemple) il peut apprécier directement un petit nombre de probabilités canoniques $P\left(E_{0}\right)$ et extrapoler ou interpoler les autres par une formule.

\section{Principes de comportement cohérent.}

Maintenant une loterie pourra représenter soit une loterie canonique soit un acte réel dont la conséquence (le prix) peut dépendre de l'occurrence d'un événement "réel".

\section{Troisième hypothèse.}

Les relations de préférence ou d'indifférence entre les loteries doivent être transitives : si le décideur préfère la loterie $A$ à $B$ et s'il préfère $B$ à $C$ ou est indifférent entre $B$ et $C$ alors il doit préférer $A$ à $C$.

\section{Quatrième hypothèse.}

$\mathrm{Si}$ des prix d'une loterie sont remplacés par d'autres prix tels que le décideur est indifférent entre chaque nouveau prix et l'ancien qui lui correspond, alors il sera aussi indifférent entre les loteries originelle et modifiée.

\section{Cinquième hypothèse.}

Si deux loteries $L$ et $L^{\prime}$ sont telles que leur résultat est annulé si un certain événement $E_{0}$ ne survient pas et si le décideur préfère $L$ à $L^{\prime}$, alors (et seulement dans ce cas) $L$ est préférée à $L^{\prime}$ conditionnellement à l'occurrence de $E_{0}$.

\section{Théorèmes principaux résultant des 5 hypothèses.}

\section{Premier théorème.}

Lès probabilités canoniques $P\left(E_{j}\right)$ affectées aux événements $E_{j}$ obéissent aux axiomes usuels du calcul des probabilités, c'est-à-dire :

$$
\begin{aligned}
& P\left(E_{j}\right) \geqslant 0 \text { quel que soit } j \\
& \text { si } E \text { est certain } P(E)=1 \\
& \text { si } E_{1} E_{2} \ldots E_{k} \text { sont mutuellement exclusifs : } \\
& \begin{array}{r}
P\left(E_{1} \text { ou } E_{2} \text { ou } \ldots \text { ou } E_{k}\right) \\
\quad=P\left(E_{1}\right)+P\left(E_{2}\right)+\ldots+P\left(E_{k}\right)
\end{array}
\end{aligned}
$$

Ce résultat justifie a posteriori le nom de probabilités donné aux $P\left(E_{j}\right)$.

\section{Deuxième théorème.}

Soit $a$ un acte quelconque et $E_{1} E_{2} \ldots E_{K}$ l'ensemble des $K$ événements possibles supposés mutuellement exclusifs ; soient $H_{1} H_{2} \ldots H_{K}$ les conséquences de $a$ relatives à ces événements et:

$$
\bar{\pi}=\sum_{j} P\left(E_{j}\right) \pi\left(H_{j}\right)
$$

Alors le décideur est indifférent entre l'acte $a$ et une loterie canonique donnant une probabilité canonique $\bar{\pi}$ à $\bar{H}$ et $1-\bar{\pi}$ à $\underline{H}$.

Il résulte de ce théorème important que l'ordre de préférence des actes (ou décisions) peut être donné par l'ordre des valeurs numériques de $\bar{\pi}$. Si on appelle $\pi\left(H_{j}\right)$ l'utilité de la conséquence $H_{i}$, on voit que la méthode de choix des décisions revient à maximiser l'espérance mathématique des utilités (formule (1.A) pondérées par les probabilités "subjectives" $P\left(E_{j}\right)$.

\section{Troisième théorème.}

Soit $E^{\prime}$ et $E^{\prime \prime}$ deux événements tels que : $P\left(E^{\prime}\right)>0$ et supposons que le décideur veuille apprécier la probabilité de l'événement combiné $\left(E^{\prime}\right.$ et $\left.E^{\prime}\right)$. Le théorème suivant fournit la réponse :

Soit $L_{E}$, et $L$ deux loteries dont le prix est nul à moins que $E^{\prime}$ survienne. Dans le cas où $E^{\prime}$ survient, $L_{E}$ paiera $\bar{H}$ si $E^{\prime \prime}$ survient et $H$ dans le cas contraire pendant que $L$ donnera une probabilité canonique $p$ à $\bar{H}$ et $1-p$ à $\underline{H}$. Alors le décideur est indifférent entre $L_{E}$ et $L$ si et seulement si :

$$
p=\frac{P\left(E^{\prime} \text { et } E^{\prime \prime}\right)}{P\left(E^{\prime}\right)}
$$

$p$ se définit comme la probabilité conditionnelle à $E^{\prime}$ fixé : $p\left(E^{\prime \prime} / E^{\prime}\right)$ et la relation (22) est en fait la règle des probabilités composées :

$$
\left.P\left(E^{3} \text { et } E^{\prime \prime}\right)=P\left(E^{\prime}\right) \cdot P / E 3 / E^{\prime}\right)
$$

C'est de cette règle qu'est tiré immédiatement le théorème de Bayes. 